\title{
Principles of topical therapy
}

The availability of topical agents for treatment of acne is as wide and variable as the manifestations of acne itself. As with systemic therapy, the choice of agent for topical therapy depends on the clinical presentation and the general principles of treatment.

The general principles of treatment are based on: decreasing sebaceous gland secretion; correcting ductal hypercornification; decreasing $P$. acnes population; and producing an anti-inflammatory effect. ${ }^{[1]}$ At present, there is no topical therapy available that has all the above properties and, therefore, one has to choose more than one topical agent to achieve the desired result.

The choice of topical agent depends on two main variables: clinical condition - inflammatory or noninflammatory - and the pharmacology of the agent - antimicrobial, keratolytic, or anti-inflammatory. Before choosing the agent, a good examination of the skin is required to determine the type of acne and the extent of involvement. Due consideration needs to be given to the age of the patient, the skin type, and existence of special features such as pregnancy, steroid abuse, or skin irritation.

The pharmacology of each potential topical agent needs to be evaluated for its formulation (lotion, cream, or gel), mechanism of action, frequency of application, expected duration of therapy, and concomitant use of adjunctives.

\section{GENERAL ADVICE}

Patient instruction is paramount in achieving the best potential of the chosen topical treatment. The patient must be made to understand that topical treatment is necessary not only during the active phase of acne but also during the maintenance and preventive phase. This involves regular application of topical agents over a prolonged period. The methodology of the application has to be explained to the patient to maximize compliance as the therapeutic outcome depends on correct and regular application.

Spot versus total area application: The patient must be educated to understand that the logic of most topical treatments is to prevent development of new lesions, and as the precursor lesion of acne - the microcomedo - is invisible, it is essential to apply the topical agent to the entire susceptible area.

Frequency of application: Topical antimicrobials are best applied 2-3 times per day while topical retinoids and benzoyl peroxide (BPO) be limited to once daily application to avoid excessive drying or irritation.

Time of application: Retinoids are best applied at night to avoid occasional photosensitivity. For other topical agents, there is flexibility. Slotting of topical agents requires individualizing. Any latitude in this matter, if possible, should be explained to the patient. This will ensure adherence during vacations, travel, and other fluctuations in daily routine.

Methodology: The patient is instructed to clean the target area with the chosen cleanser (preferably a gentle cleanser). After the skin has dried, it is gently stretched and the topical agent is applied as a thin film over the entire area. The necessity of percutaneous absorption is explained to the patient, and the importance of gentle rubbing to achieve this is emphasized. The quantity to be applied should be discussed. In general, a quantity that covers the target area and gets absorbed with gentle rubbing in 30 seconds is deemed adequate. However, the adequate quantity of the topical product depends on its viscosity and the spread-ability and, therefore, some trial and error, and some learning is called for. Topical retinoid treatment is a special case and warrants gentle induction. It is advisable to start with short contact - 15-30 minutes - for 2-4 weeks, and then switch to overnight application. ${ }^{[2]}$

Delivery system: The cream base is preferred for dry skin and the gels are preferred for oily skin. ${ }^{[3,4]}$ Lotions are preferred when treating large areas such as the trunk. Soaps and face washes containing antiacne agents may be used as adjunctives.

Special precautions: Infants and neonates, and patients with sensitive skin may be treated with creams or gels with lower concentrations. Retinoids should be avoided in pregnant women. Concomitant use of 
dissimilar topical and systemic antibiotics should be minimized or, better, avoided..$^{[5]}$

\section{REFERENCES}

1. Cunliffe W, Gollnick HPM. Acne: Diagnosis and management. London: Martin Dunitz; 2001. p. 107-14.

2. Batra SR. Acne. In: Arndt KA, Hsu JT, editors. Manual of dermatologic therapeutics $7^{\text {th }}$ ed. Philadelphia: Lippincott Williams and Wilkins; 2007. p. 3-18.

3. Chivot M. Treatment of mild to moderate acne. Ann Dermatol Venereol 2004;130:132-5.

4. Arndt KA, Bowers KE. Manual of dermatologic therapeutics with essentials of diagnosis. $6^{\text {th }}$ ed. Philadephia: Lippincott, Williams and Wilkins; 2002. p. 3-20.

5. Gollnick H, Cunliffe W, Berson D, Dreno B, Finlay A, Leyden JJ, et al. Management of acne: A report from a global alliance to improve outcomes in acne. J Am Acad Dermatol 2003;49:S1-38. 